\title{
dol $10.4067 / 50718-48082021000100039$

La medición de la felicidad a través del Test de
Asociación Implícita y su relación con la
PSICOLÓGICA
$\frac{\text { depresión y el bienestar autoinformados }}{\text { ISSN: } 0718-4808}$

Diego Gómez-Baya'

(iD) 0000-0003-4651-0439

diego.gomezedpee.uhu.es

José Antonio Muñiz-Velázquez ${ }^{2}$

(iD) 0000-0003-4518-3624

jamunizeuloyola.es

1 Universidad de Huelva. Huelva, España. Depto. de Psicología, Social, Evolutiva y de la Educación

2 Universidad Loyola. Sevilla, España. Depto. de Comunicación y Educación.

\section{Resumen:}

La evaluación del bienestar subjetivo y psicológico ha alcanzado considerable evidencia científica, principalmente a través de diferentes pruebas de autoinforme. Sin poner en cuestión este tipo de medición, lo cierto es que estas mediciones presentan la desventaja de estar basadas en declaraciones subjetivas y manipulables conscientemente por parte del sujeto. El objetivo de este trabajo es presentar un diseño bajo el paradigma del Test de Asociación Implícita de Greenwald y Farnham (2000) para aplicarlo a la medición implícita del bienestar subjetivo y psicológico. Así, se realizó un estudio transversal descriptivo con una muestra de 327 adultos $(51,7 \%$ hombres; $M=38,69, D T=11,98)$, que completaron un Test de Asociación Implícita para la medida objetiva del bienestar psicológico, y dos medidas autoinformadas para evaluar por un lado la felicidad y por otros posibles síntomas depresivos. Los resultados mostraron una buena fiabilidad del Test de Asociación Implícita, así como asociaciones débiles con las medidas de autoinforme. Una asociación implícita más fuerte con los afectos positivos que con los afectos negativos estuvo relacionada con una mayor felicidad subjetiva y menos síntomas depresivos. Estos resultados sugieren la posibilidad de completar la evaluación del bienestar usando medidas objetivas.

Palabras claves: prueba de asociación implícita; felicidad; depresión; procesos implícitos, afecto.

\section{Abstract:}

The assessment of subjective and psychological well-being has reached considerable scientific evidence, mainly through different self-report tests. Without questioning this type of measurement, the fact is that these measurements have the disadvantage of being based on subjective statements that can be consciously manipulated by the subject. The aim of this work is to present a new instrument following the paradigm of the Implicit Association Test by Greenwald and Farnham (2000) in order to provide the implicit measurement of subjective and psychological well-being. Thus, a descriptive cross-sectional study was carried out with a sample of 327 adults $(51.7 \%$ men; $M=38.69, S D=11.98)$, who completed an Implicit Association Test for the objective measurement of psychological well-being, and two selfreported measures to assess happiness and depressive symptoms. The results showed good reliability of the Implicit Association Test, as well as weak associations with the self-report measures. A stronger implicit association with positive affects than with negative affects was related to higher subjective happiness and fewer depressive symptoms. These results suggest the possibility of completing the well-being assessment using objective measures.

Keywords: implicit association test; happiness; depression; implicit; affect. 


\section{Introducción}

Hasta la fecha, la evaluación de la felicidad hedónica y eudaimónica del ser humano ha alcanzado considerable evidencia científica, principalmente a través de diferentes escalas y cuestionarios psicológicos. De entre la gran cantidad de estos instrumentos de autoinforme que han ido apareciendo en las últimas décadas, muchos merecerían ser destacados. Tal es el caso de la Escala de Satisfacción Vital (Satisfaction With Life Scale; SWLS) de Diener, Horwitz y Emmons (1985), el Cuestionario de Emociones de Fordyce (Fordyce, 1988), las Escalas de Afectos Positivos y Negativos (Positive And Negative Affect Scales; PANAS) de Watson, Clark y Tellegen (1988), la Escala de Felicidad Subjetiva (Subjective Happiness Scale; SHS) de Lyubomirsky y Lepper (1999), El Cuestionario de Felicidad de Oxford (Hill y Argyle, 2002), el Método de Reconstrucción del Día (Day Reconstruction Method; DRM) por Kahneman, Krueger, Schkade, Schwarz y Stone (2004), el Inventario de la Felicidad Auténtica (Authentic Happiness Inventory; AHI) de Peterson (2005), la Escala de Bienestar Mental de Warwick-Edimburgo (Warwick-Edinburgh Mental Well-Being Scale- WEMWBS) de Tennant et al. (2007), La Escala de Florencimiento (Flourishing Scale; FS) de Diener et al. (2009), y el Índice de Felicidad de Pemberton (Pemberton Happiness Index; PHI) de Hervás y Vázquez (2013), entre otros muchos.

Todas estas escalas han sido validadas psicométricamente, y como decían De las CuevasCatresana y González de Rivera-Revuelta (1992) en relación con el conjunto de escalas de autoinforme, suponen un método directo de evaluar las respuestas cognitivas y la experiencia subjetiva del individuo. Pero lo cierto es que, aunque cuenten con mecanismos para mitigar muchos de los sesgos voluntarios e involuntarios en las respuestas, así como otras variables extrañas, siguen presentando ciertos inconvenientes (Gómez-Benito, Hidalgo y Guilera, 2010). Inconvenientes que parten del hecho de ser mediciones basadas en declaraciones subjetivas, explícitas y conscientes de los participantes que son preguntados. Cuando una persona responde un cuestionario lo hace a través de procesos cognitivos que son explícitos y en buena medida bajo su control (Díaz, Horcajo y Blanco, 2009; Kurdi et al., 2019). Por lo tanto, estamos hablando de respuestas siempre manipulables de manera consciente, o inconsciente. Asimismo, pueden aparecer sesgos causados, por ejemplo, por la deseabilidad social, o debido al engaño o falsificación, e incluso al autoengaño, que haría diferenciar entre una felicidad más objetiva de aquella felicidad auto-percibida (Muñiz-Velázquez, Gómez-Baya y López-Casquete, 2017; Van de Mortel, 2008; Warriner, Shore, Schmidt, Imbault y Kuperman, 2017; Young, Kashdan, y Macatee, 2014). Caputo (2017) mostró cómo las medias autoinformadas de bienestar se encontraban correlacionadas de forma moderada con escalas de deseabilidad social. Así, la deseabilidad social explicaba una varianza del bienestar entre el $3 \%$ y el $6 \%$, tras controlar las variables sociodemográficas. Como ya apuntaron Diener, Sandvik, Pavot y Gallagher (1991), es necesario controlar algunos posibles artefactos en las medidas de autoinforme, como la deseabilidad social, el actual estado de ánimo, o las creencias morales sobre la felicidad. Para superar estas limitaciones, Diener (1994) recomendó un abordaje multimétodo, para una descripción más comprehensiva del bienestar, incluyendo componentes faciales, fisiológicos, motivacionales, conductuales y cognitivos. 
Con todo, es cierto que las escalas psicométricas dan mucha luz, pero ¿en qué medida estamos seguros de poder encontrar la medida de nuestro verdadero bienestar sin los obstáculos antes descritos? Dejando aparte en este momento las posibilidades crecientes, pero también costosas de las técnicas de observación neurocientífica (Alexander et al. 2021; Zevnik, 2014) tales como el electroencefalograma, eye-tracking, imágenes de resonancia magnética funcional, actividad electro-dérmica, la tomografía axial computarizada, etc., la cuestión es poder observar la felicidad del individuo sin su propia automediación subjetiva, evitando por tanto sesgos y, al mismo tiempo, utilizando indicadores más allá de escalas numéricas limitadas. Así, para esta sustitución del uso de escalas, podemos encontrar algunos ejemplos recientes de métodos experimentales que han intentado medir diferentes variables cercanas al amplio constructo de la felicidad. Por ejemplo, el utilizado por Sánchez, Vazquez, Gomez y Joormann (2014), el llamado Karolinska Directed Emotional Faces (KDEF), con el que observar la reparación emocional después de un procedimiento de inducción de estado de ánimo a través de la exposición a ciertas caras. Asimismo, existe el ejemplo de una interesante herramienta de control deslizante con la que Warriner et al. (2017) intentaron medir la evaluación afectiva de las palabras a través de las distancias encontradas entre un conjunto de palabras y un maniquí movido por los sujetos que los representaban. Otros estudios han abordado la evaluación del bienestar subjetivo mediante la técnica de redes semánticas naturales, que permite identificar cuáles son los conceptos o palabras más relevantes o centrales para definir la felicidad. En esta línea, Flores-Kanter, Muñoz-Navarro, y Medrano (2018) concluyeron en una muestra de adultos en Argentina que los participantes concibieron la felicidad en términos tanto hedónicos como eudaimónicos, y que aquellos que presentaban más felicidad subjetiva autoinformada presentaban una idea de la felicidad con conceptos más eudaimónicos. Denegri, García, y González (2015) mostraron, en una muestra de adultos en Chile, que los elementos más vinculados a la felicidad y la satisfacción vital eran conceptos relacionados con la familia, los amigos y el amor, así como otros conceptos relativos al trabajo, las metas profesionales y las actividades recreativas.

Pero sin lugar a duda, al hablar de las herramientas más prometedoras de evaluación más allá de las escalas, debemos destacar el Test de Asociación Implícita (Implicit Association Test o IAT) como una de las formas más convincentes y con más soporte empírico hasta la fecha (Horcajo et al., 2014; Irving y Smith, 2020; Kurdi et al., 2019). El paradigma IAT fue desarrollado por Greenwald y Farnham (2000) para medir las diferencias individuales en la cognición implícita en el campo de la psicología social: actitudes, cambio de actitudes, prejuicios sociales, racismo, orientación sexual, autoestima, entre otros constructos psicológicos. El IAT detecta la fuerza de la asociación automática de una persona entre representaciones mentales de objetos, palabras o conceptos en la memoria, en términos de tiempo de reacción en una tarea simple. Por ejemplo, una tarea como presionar una tecla en el teclado de la computadora cuando aparecen ciertos estímulos en la pantalla, estímulos incluso tan simples como puntos y líneas que forman algo similar a las caras, con los cuales se observa, por ejemplo, la asociación implícita con las emociones humanas (Salgado-Montejo, Salgado, Alvarado y Spence, 2016). Las medidas de IAT han mostrado de manera consistente su validez predictiva y de constructo (Greenwald, Poehlman, Uhlmann, y Banaji, 2009; Nosek, Greenwald, 
y Banaji, 2005). También ha obtenido evidencia favorable la forma abreviada del IAT (Sriram y Greenwald, 2009). Hofmann, Gawronski, Gschwendner, Le, y Schmitt (2005) realizaron un metaanálisis de 126 estudios, en los que encontraron que las medidas implícitas basadas en este paradigma mostraban asociaciones pequeñas con las medidas de autoinforme, si bien la mitad de esta varianza se podría atribuir a otras variables moderadoras. Así pues, una medida más comprehensiva del bienestar pasa por incluir evaluaciones tanto de aspectos implícitos como explícitos, integrando las medidas desde el paradigma IAT con las medidas de autoinforme.

Es cierto que existe cierta controversia sobre lo que realmente mide (Rohner y Ewers, 2016), pero cada día es más la investigación que avala su validez y sus propiedades psicométricas (Jost, 2019). Hoy en día, el modelo IAT se usa no solo en psicología social, sino también en otros campos que van desde la investigación clínica (Burgess, Nugent, Hunt y Eslami, 2019; Rath, et al., 2018; da Victoria, Nascimento y Fontenelle, 2011), a la psicología política (Sánchez, 2012), el estudio de la comunicación y del consumo de medios (Garín et al. 2013), o del consumo en general (Gattol, Saaksjarvi y Carbon, 2011; Maison, 2016), por ejemplo. También dentro de la psicología positiva, la medición implícita de la felicidad y el bienestar ya se ha abierto un cierto camino en los últimos años. Los mismos Greenwald y Farnham (2000) midieron la autoestima y el autoconcepto. Pero un primer ensayo de IAT para evaluar el bienestar subjetivo, principalmente hedónico, fue llevado a cabo por Walker y Schimmack (2008) en la Universidad de Toronto con una muestra de 150 estudiantes. También Young, Kashdan, y Macatee (2014) probaron con una muestra de 140 adultos, un IAT para estudiar las fortalezas humanas, buscando la importancia de equilibrar las fortalezas distintivas o motivadoras en comparación con aquellas fortalezas no distintivas en la predicción de un mayor bienestar. Además, Park, Uchida y Kitayama (2016) utilizaron el IAT para comparar las diferencias entre los estadounidenses, los europeos y los japoneses $(n=66)$ con respecto a la importancia de la independencia frente a la interdependencia para su autodefinición, las emociones y la felicidad.

También Deng, Ding, Cheng y Chou (2016) han utilizado IAT para medir las actitudes implícitas hacia la felicidad en sí misma, en concreto las actitudes implícitas hedónicas hacia la felicidad y la experiencia de emociones mixtas durante eventos emocionales agradables ( $n=$ 119). Estos autores subrayaron el papel de las diferencias culturales y subculturales, en su caso entre los chinos Han y los chinos de Mongolia. Con una muestra más grande ( $n=400$ ), Riediger, Wrzus y Wagner (2014) también utilizaron el IAT para buscar las asociaciones implícitas de felicidad de los participantes con la amabilidad, junto con la motivación hedónica y experiencias simultáneas de experiencias positivas y positivas. Costantini et al. (2019) también usaron una prueba de asociación implícita para medir la orientación positiva $(\mathrm{OP})$. Es un constructo cercano a la autoestima, la satisfacción con la vida y el optimismo, siendo definido como una "predisposición básica que consiste en sentirse digno de consideración, con una vida digna y un futuro prometedor" (Costantini et al., 2019, p. 1). Con una muestra de 254 estudiantes italianos, validaron su OP-IAT con muy buenas propiedades psicométricas. 
Asimismo, el uso del IAT en español en el estudio de constructos relacionados de una manera u otra con el bienestar, es una realidad. Tal es el reciente caso, por ejemplo, de Corral-Frías, Lucas, Corral-Frias, Corral-Verdugo y Tapia (2019), quienes observaron en una muestra de estudiantes mexicanos la relación entre la actitud hacia la diversidad, el conservacionismo medioambiental y el bienestar. Asimismo, unos años antes, Díaz, Horcajo y Blanco (2009) complementaron sus mediciones explícitas de bienestar con una adaptación propia del Implicit Association Test (IAT) al que llamaron Medida Implícita de Bienestar General (MIBG). En sus estudios encontraron resultados moderadamente satisfactorios en cuanto a la potencia observacional de la herramienta, abriendo así una vía de estudio que, sin embargo, ha quedado en buena medida pendiente de transitar.

Así, profundizar en los temas tan amplios de hedonia y eudaimonia, también con muestras de individuos más grandes y variadas, es una necesidad para explorar y demostrar la capacidad de la IAT para iluminar sin filtros la observación científica de nuestra felicidad y bienestar tanto subjetivo como psicológico, ayudando en este sentido al trabajo realizado hasta la fecha con los autoinformes. Hasta la fecha la mayor parte de estudios desde el paradigma IAT se han realizado con población anglosajona y se requieren más evidencias con población hispanohablante. Algunos trabajos han examinado el cambio de actitudes ante mensajes persuasivos (Briñol, Horcajo, Becerra, Falces y Sierra, 2002), las actitudes hacia las mujeres (Cárdenas, González, Becerra, Falces y Sierra, 2009), hacia la diversidad sexual (Cárdenas y Barrientos, 2008), hacia la violencia (Garín, Huenchuleo, Leal, Muñoz y Rehbein, 2013) o hacia la naturaleza (Olivos y Aragonés, 2013). Más evidencia desde este paradigma es necesaria en muestras hispanohablantes para medir el bienestar psicológico, con sólo las evidencias de los trabajos de Corral-Frías et al. (2019) y Díaz et al. (2009).

\section{Objetivos e hipótesis}

Siguiendo el camino abierto por Walker y Schimmack (2008), y tímidamente por Díaz, Horcajo y Blanco (2009) dentro de la observación implícita del bienestar subjetivo, el objetivo principal del presente trabajo es aplicar el mencionado paradigma del Test de Asociación Implícita de Greenwald y Farnham (2000) al campo de la medición del bienestar subjetivo. Para examinar la validez de constructo se estudiará asimismo la relación con medidas autoinformadas tanto de bienestar psicológico como de malestar (en este caso, sintomatología depresiva), que ya han bien trabajadas en la literatura previa.

Para ello, nuestros objetivos específicos e hipótesis fueron los siguientes:

1 Construir una nueva prueba de asociación implícita para evaluar las relaciones entre el Yo y los afectos positivos y negativos, a partir de lo cual se configura la Hipótesis 1: se espera desarrollar un IAT fiable y válido para evaluar, de manera implícita y por lo tanto con menos interferencias, el bienestar subjetivo de los individuos.

2. Analizar hasta qué punto las medidas implícitas permiten explicar medidas autoinformadas o explícitas de la felicidad y la depresión, a partir de lo cual se configura la Hipótesis 2: Se espera encontrar asociaciones moderadas entre las medidas implícitas y las evaluaciones explícitas de la felicidad y la depresión, como señalaron Walker y Schimmack (2008), y como en cierta medida encontraron Díaz, Horcajo y Blanco (2009). 


\section{Método}

\section{Diseño general del estudio y procedimiento de recogida de datos}

Se ha seguido un diseño transversal y descriptivo, en el que los participantes completaron de forma anónima y online los instrumentos y las tareas de evaluación que se describen a continuación. Anteriormente, se llevó a cabo un estudio piloto, para analizar las propiedades psicométricas de los instrumentos y asegurar que no hubiera ningún tipo de confusión o malestar, evaluando a una muestra de 190 adultos con características similares a la muestra incluida en el trabajo de campo. Este trabajo de investigación respetó todos los principios incluidos en la Declaración de Helsinki sobre investigación con seres humanos, y todos los individuos participaron voluntariamente, fueron informados de las características de este estudio y dieron su consentimiento informado. El contacto inicial se realizó a través de un correo electrónico solicitando la participación a cambio de puntos que se intercambiarán por varios premios dentro del sistema de panel de consumidores. Después del consentimiento informado, una semana después, cada participante recibió un correo electrónico con un enlace que lo llevaría a la página donde se implementó el instrumento de evaluación. Los datos se registraron en la plataforma online y posteriormente se transformaron en una hoja de cálculo, registrando la hora de inicio en la tarea y la hora de finalización de esta. En promedio, los participantes realizaron la tarea en aproximadamente 20 minutos, presentando omisiones muy bajas (por debajo del 0,5\%).

\section{Participantes}

La muestra de participantes estuvo compuesta por un total de 327 adultos (51,7\% hombres), con edades comprendidas entre los 18 y los 65 años $(M=38,69, D T=11,98)$, seleccionados al azar dentro de un panel de consumidores que eran adultos españoles, siguiendo un muestreo estratificado realizado por una empresa externa. En cuanto al estado civil, el 38,2\% era soltero/a, el 56,3\% estaba casado/a o vivía en pareja, el 5,2\% estaba separado/a o divorciado/a, y el 0,3\% eran viudo/a. Con respecto al nivel académico, el 0,3\% no tenía estudios, el 0,3\% completó la educación primaria, el 5,2\% completó la primera etapa de la educación secundaria, el $23,2 \%$ completó la educación secundaria o tenía formación profesional media, el 20,2\% tenía un bachillerato o tenía formación profesional superior, y el 50,8 tenían estudios universitarios. Respecto a la situación laboral, el 60,6\% trabajaba actualmente, el 4,9\% buscaba su primer empleo, el 4,6\% tenía una jubilación anticipada, el $10,7 \%$ eran estudiantes, el $18 \%$ estaba desempleado/a y el 1,2\% trabajaba en casa. Finalmente, con respecto al nivel de ingresos anuales, el 16,2\% declaró 6,000€ o menos, el $19,2 \%$ tuvo entre $6,000 €$ y $15,000 €$, el $24.1 \%$ tuvo entre $15,001 €$ y $30,000 €$, el $17,3 \%$ presentó ingresos por encima de $30,001 €$, y hasta el $23,2 \%$ prefirió no responder.

\section{Instrumento}

Además de medir algunas variables sociodemográficas, como el género, la edad, el estado civil, el nivel de estudios, la situación laboral y el nivel de ingresos, se administraron las siguientes pruebas: 


\section{Medidas explícitas}

Síntomas depresivos. Se empleó el Major Depression Inventory (MDI) (Olsen, Jensen, Noerholm, Martiny y Bech, 2003) en su adaptación al castellano (Nebreda y Aliaga, 2003). Este autoinforme sobre el estado de ánimo desarrollado la Organización Mundial de la Salud presenta 10 ítems representados con 12 cuestiones (los ítems 8 y 10 presentan a su vez dos sub-ítems cada uno, a y b) en las que se pide al encuestado que informe con qué frecuencia ha experimentado diferentes síntomas, tras el siguiente enunciado: "Las siguientes preguntas hacen referencia a su estado de ánimo en las últimas dos semanas. Por favor marque la casilla que mejor describa cómo se ha sentido usted". Se presenta una escala tipo Likert de seis opciones de respuesta: "todo el tiempo", "la mayor parte del tiempo", "poco más de la mitad del tiempo", "poco menos de la mitad del tiempo", "ocasionalmente" y "nunca". Para el cálculo de la puntuación total, se realiza la suma de las respuestas en cada uno de los 10 ítems, codificados de 0 a 5 desde "nunca" a "todo el tiempo". En esta suma, respecto a los ítems 8 y 10 se incorpora el sub-ítem $(a \circ$ b) que tenga la puntuación más alta. Así, una mayor puntuación indica una mayor presencia de síntomas depresivos. Este inventario puede ser utilizado como una medida de severidad en los síntomas depresivos, tomando puntuaciones desde 0 a 50, de manera que puntuaciones entre 20-24 representan depresión leve, puntuaciones entre 25-29, depresión moderada, y valores por encima de 30, depresión severa (Olsen, Jensen, Noerholm, Martiny y Bech, 2003). También, puede ser empleado como instrumento para el diagnóstico de trastorno depresivo mayor siguiendo directrices de ICD-10 y DSM-IV (Bech, Rasmussen, Olsen, Noerholm y Abildgaard, 2001). En este trabajo, la escala ha presentado una excelente fiabilidad por consistencia interna, alcanzando un valor a de Cronbach de 0,92.

Felicidad subjetiva. Se utilizó el Pemberton Happiness Index, en su versión española validada por Hervas y Vazquez (2013). Esta escala autoinformada recoge, por un lado, 11 ítems relacionados con diferentes dominios del bienestar recordado (general, hedónico, eudaimónico y social) que se responden siguiendo una escala tipo Likert de 11 puntos, desde 0 (totalmente en desacuerdo) hasta 10 (totalmente de acuerdo). En concreto, los ítems 1 y 2 miden bienestar general, los ítems 3-8, evalúan bienestar eudaimónico, los ítems 9 y 10 miden bienestar hedónico, y el ítem 11, el bienestar social. Para el cálculo de la puntuación total en bienestar recordado se produce la suma de la respuesta en cada ítem, recodificando el ítem 10. Así, las puntuaciones oscilan en un rango entre 0 y 110, indicando una puntuación más elevada un mayor nivel de bienestar recordado.

En el presente estudio, la escala de bienestar recordado presentó una notable fiabilidad por consistencia interna, alcanzando un valor a de 0,85. Por otro lado, la escala presenta 10 indicadores de bienestar experimentado, que reflejan eventos emocionales positivos y negativos que hayan ocurrido el día anterior, y que se responden con una escala dicotómica Sí o No. Los indicadores 1, 3, 5, 7 y 9 reflejan experiencias positivas, mientras que los ítems 2, 4, 6, 8 y 10 muestran experiencias negativas. En los indicadores positivos las opciones de respuesta se codifican como Sí (1) y No (0), mientras que en los indicadores negativos la codificación es inversa (Sí valdría 0 y No, 1). Así, estos diez ítems pueden dar como resultado 
una única puntuación de 0 (ninguna experiencia positiva y 5 experiencias negativas) a 10 (5 experiencias positivas y ninguna experiencia negativa). Para obtener la puntuación total de felicidad se suma la puntuación en bienestar recordado con la puntuación en bienestar experimentado. Cuando se calcula la fiabilidad de los ítems de bienestar recordado y se incluye la puntuación total en bienestar experimentado, componiendo la medida de total felicidad, se obtiene un valor a de 0,86.

\section{Medidas implícitas}

Asociaciones automáticas del yo con afectos positivos y negativos. Se ha diseñado una prueba de asociación implícita siguiendo el procedimiento detallado por Greenwald y Farnham (2000) con vistas a medir el tiempo de reacción empleado en asociar al yo afectos positivos y negativos, y el tiempo de reacción que se tarda en no asociar al yo afectos positivos y negativos. Así, esta tarea quiere analizar la relación entre dos grupos de palabras, por un lado, pronombres que hacen referencia al yo (yo, mi, mío, conmigo) o a no-yo (ellos, sus, otros, suyos), y, por otro lado, adjetivos que hacen referencia a afectos positivos (satisfecho, feliz, alegre, contento) o negativos (triste, molesto, apenado, deprimido). La prueba está compuesta por 7 bloques, de los cuales 3 (los bloques 1, 2 y 5) son de prueba y 4 bloques son objeto de análisis (los bloques 3, 4, 6 y 7), siguiendo las indicaciones de Greenwald, Nosek y Banaji (2003). En cada uno de los bloques se presentarán aleatoriamente en el centro de la pantalla palabras (en color negro sobre fondo blanco) que el participante ha de clasificar como miembro de la categoría que se describe en la parte superior izquierda de la pantalla (pulsando la tecla "q" del teclado de su ordenador) o de la categoría indicada en la parte superior derecha de la pantalla (pulsando la tecla "p" del teclado).

En el bloque 1, se presentan en el centro de la pantalla, secuencial y aleatoriamente, los 8 pronombres personales (yo, mi, mío, conmigo, ellos, sus, otros, suyos), mientras que en la parte superior izquierda se indica la categoría "Yo" y en la parte superior derecha, la categoría "No Yo". El participante ha de pulsar la letra "q" si el pronombre hace referencia a la categoría "Yo" o la letra "p" si pertenece a la categoría "No Yo". En el bloque 2, se presentan aleatoriamente los 8 adjetivos que representan estados afectivos (satisfecho, feliz, alegre, contento, triste, molesto, apenado, deprimido) y el participante ha de indicar si corresponden a la categoría "Positivo", pulsando la letra "q" o a la categoría "Negativo", pulsando la letra "p". El tercer bloque presentará aleatoriamente las 16 palabras presentadas en los bloques 1 y 2, es decir, los 8 pronombres y los 8 adjetivos, y el participante ha de elegir entre dos categorías compuestas, a la izquierda "Yo-Positivo" y a la derecha "No Yo- Negativo". Si la palabra es un pronombre de la primera persona del singular o si la palabra es un adjetivo que hace referencia a un estado afectivo positivo, el participante ha de pulsar la letra "q" para indicar la categoría de la izquierda, en cambio, si la palabra no es un pronombre de la primera persona del singular o es un adjetivo que describe un estado afectivo negativo, el participante tendrá que pulsar la letra "p", señalando así la categoría de la derecha. El cuarto bloque presenta similar estructura que el tercero. 
En el quinto bloque, vuelven a presentarse los 8 pronombres solo que esta vez las categorías aparecen intercambiadas, de manera que el participante ha de pulsar la letra "q" si el pronombre hace referencia a la categoría "No Yo" o la letra " $p$ " si pertenece a la categoría "Yo". En los bloques sexto y séptimo, se presentan en cada uno al azar, una a una, las 16 palabras (los pronombres y los adjetivos), y el sujeto ha de discriminar entre dos categorías compuestas, a la izquierda "No Yo-Positivo", pulsando la letra "q", y a la derecha "YoNegativo", pulsando la letra "p". En cada ensayo, se computará el tiempo de reacción desde la aparición de la palabra hasta que se pulsa la tecla correspondiente. Se calcularon cuatro sumas de tiempos de reacción, el tiempo empleado en asociar al Yo estados afectivos positivos, el tiempo empleado en asociar al Yo estados afectivos negativos, el tiempo empleado en asociar a No Yo estados afectivos positivos, y el tiempo empleado en asociar a No Yo estados afectivos negativos.

A partir de estas sumas de tiempo de reacción se calcularon a su vez las diferencias, siguiendo el modelo canónico de Greenwald, Nosek y Banaji (2003). En primer lugar, se calculó la diferencia entre el tiempo de reacción en la asociación Yo-Afecto Negativo menos el tiempo de reacción Yo-Afecto Positivo, dividida entre las desviaciones típicas. En segundo lugar, se calculó la diferencia entre el tiempo de No Yo- Afecto Positivo menos No Yo- Afecto Negativo, entre las correspondientes desviaciones típicas. Se estima que las personas que se sientan mejor tendrán más facilidad (y por tanto un menor tiempo de reacción) para asociar al Yo afectos positivos y les costará más tiempo asociar al Yo afectos negativos. Además, las personas que se sientan mejor tardarán menos en asociar a No-Yo afectos negativos, pero les resultará más difícil asociar a No-Yo afectos positivos. Así, una mayor puntuación en las diferencias calculadas indica una mayor asociación implícita de afectos positivos y menor asociación de afectos negativos con el Yo.

\section{Diseño de análisis de datos}

Respecto al primer objetivo, la construcción de una prueba de evaluación de asociaciones implícitas del yo con afectos positivos y negativos, se llevaron a cabo varios análisis de datos. Tras el estudio de los estadísticos descriptivos, se procedió al estudio de la normalidad de la distribución de las variables mediante la prueba de Kolmogorov-Smirnov con la corrección de Lilliefors. En primer lugar, se analizó la fiabilidad por consistencia interna (a de Cronbach) y por dos mitades (Guttman) de los ensayos que componen cada medida implícita, y de la prueba en general. En segundo lugar, como pruebas de validez, se realizaron correlaciones bivariadas de orden cero para estudiar las relaciones entre los indicadores implícitos (tiempo de asociación Yo - Afecto Positivo, tiempo de asociación Yo - Afecto Negativo, tiempo de asociación No Yo - Afecto Positivo, tiempo de asociación No Yo - Afecto Negativo, diferencia entre las asociaciones del Yo y diferencia entre las asociaciones del No Yo) y las medidas explícitas de bienestar (felicidad recordada, felicidad experimentada y síntomas depresivos). Además, se realizaron análisis bivariantes para estudiar las diferencias en tiempos de reacción en cada medida implícita en función de los niveles extremos de felicidad y de depresión. 


\section{Resultados}

\section{Estadísticos descriptivos y análisis de fiabilidad}

En la Tabla 1 se presentan los estadísticos descriptivos (media y desviación típica), en la muestra total y por género, de todas las variables objeto de estudio. Respecto a las medidas implícitas, como puede observarse, las medias más elevadas en tiempo de reacción se presentan en las asociaciones No Yo - Afecto Positivo y Yo - Afecto Negativo, lo que nos muestra que a los participantes en este presente estudio tardan un mayor tiempo de reacción asociar al yo adjetivos que representan afectos negativos $(M=21,66, D T=19,33)$, y les cuesta más también no asociar al yo adjetivos que representan afectos positivos $(M=22,06, D T=$ 8,68). Por otra parte, los participantes afirman sentirse bastante felices $(M=83,79, D T=14,41)$, si bien presentan por término medio una puntuación en síntomas depresivos que se clasificaría dentro de la depresión leve $(M=22,51, D T=8,90)$. La prueba de KolmogorovSmirnov, con la corrección de significación de Lilliefors, alcanzó un nivel de significación de $p$ $<.001$ para todas las variables estudiadas, lo que indica que no siguen una distribución normal y resulta conveniente emplear análisis de datos no paramétricos. No se detectaron diferencias de género significativas, salvo en la asociación No Yo - Negativo, Standard $U=$ $-2.58, p=.010$, donde los hombres presentaron una media superior $(M=20,25, D T=7,86)$ que las mujeres $(M=18,31, D T=7,68)$.

Tabla 1: Estadísticos descriptivos de las asociaciones implícitas desde el paradigma IAT y las medidas de autoinforme de depresión y felicidad subjetiva

\begin{tabular}{lcccccc}
\hline & \multicolumn{2}{c}{ Hombres } & \multicolumn{2}{c}{ Mujeres } & \multicolumn{2}{c}{ Total } \\
& $M$ & $D T$ & $M$ & $D T$ & $M$ & $D T$ \\
\hline Yo-Afecto Positivo & 17,84 & 5,97 & 16,95 & 7,11 & 17,41 & 6,55 \\
Yo-Afecto Negativo & 22,46 & 9,96 & 20,76 & 8,95 & 21,66 & 19,33 \\
d Yo & 0,54 & 1,09 & 0,56 & 0,88 & 0,55 & 1,00 \\
No Yo-Afecto Positivo & 22,79 & 8,80 & 21,26 & 8,50 & 22,06 & 8,68 \\
No Yo-Afecto Negativo & 20,25 & 7,86 & 18,31 & 7,68 & 19,33 & 7,82 \\
d No Yo & 0,29 & 1,12 & 0,40 & 0,85 & 0,34 & 1,00 \\
Síntomas depresivos & 21,70 & 8,46 & 23,39 & 9,30 & 22,51 & 8,90 \\
Bienestar general & 14,17 & 3,51 & 14,19 & 3,21 & 14,18 & 3,36 \\
Bienestar eudaimónico & 45,37 & 8,09 & 46,27 & 8,89 & 45,80 & 8,49 \\
Bienestar hedónico & 11,59 & 2,77 & 11,94 & 2,86 & 11,75 & 2,82 \\
Bienestar social & 4,83 & 2,23 & 5,35 & 2,47 & 5,08 & 2,36 \\
Bienestar experimentado & 6,99 & 1,90 & 6,94 & 1,99 & 6,97 & 1,94 \\
Bienestar recordado & 75,95 & 13,27 & 77,75 & 13,70 & 76,82 & 13,49 \\
Felicidad & 82,95 & 14,12 & 84,69 & 14,70 & 83,79 & 14,41 \\
\hline
\end{tabular}

El estudio de la fiabilidad de los ensayos que conforman la suma de tiempos de reacción de la asociación Yo-Afecto Positivo (por consistencia interna, $a=0,73$, por estudio de las dos mitades, bloques 3 y 4, Guttman = 0,72), No yo-Afecto Negativo (por consistencia interna, $a=$ 0,75 , por estudio de las dos mitades, bloques 3 y 4, Guttman = 0,73), Yo-Afecto Negativo (por 
consistencia interna, $a=0,81$, por estudio de las dos mitades, bloques 6 y 7 , Guttman = 0,81), y No yo-Afecto Positivo (por consistencia interna, $a=0,75$, por estudio de las dos mitades, bloques 6 y 7, Guttman = 0,75), mostró resultados satisfactorios. De forma global, la fiabilidad por consistencia interna de todos los ensayos componentes de los bloques 3, 4, 6 y 7, resultó excelente, mostrando un a de 0,91. El índice de Guttman de fiabilidad por dos mitades (siendo una mitad los bloques 3 y 4 , y la otra mitad los bloques 6 y 7) fue de 0,74.

\section{Correlaciones bivariadas}

En la Tabla 2 se ofrecen los resultados de las correlaciones bivariadas de Spearman entre las variables de estudio. En primer lugar, se encontró una asociación positiva entre la diferencia en las asociaciones del Yo con la diferencia en las asociaciones de No Yo, lo que quiere decir que un mayor tiempo en asociar al yo afectos negativos y un menor tiempo en asociarle afectos positivos (que evidencia una asociación implícita más fuerte del Yo con los afectos positivos) está relacionado con un mayor tiempo para asociar a No Yo afectos positivos y un menor tiempo para asociarle afectos negativos (que evidencia una asociación implícita de No Yo con los afectos negativos). Además, dado que la diferencia del Yo se calcula en base a la diferencia entre la asociación Yo - Afecto Negativo menos Yo - Afecto Positivo, va a mostrar una correlación positiva con la asociación Yo - Afecto Negativo y una correlación negativa con Yo - Afecto Positivo. Igualmente, la diferencia del No Yo viene a mantener una correlación positiva con No Yo - Afecto Positivo y una correlación negativa con No Yo - Afecto Negativo.

En segundo lugar, puede observarse que los síntomas depresivos se asocian negativamente con todos los indicadores de bienestar, con la excepción del bienestar hedónico. Así, un mayor bienestar recordado y experimentado se relacionan con una menor presencia de síntomas depresivos. En cambio, el bienestar hedónico se relacionada de forma positiva y débil con los síntomas depresivos. Las medidas de bienestar general, bienestar eudaimónico, bienestar social y bienestar experimentado mantienen relaciones positivas entre sí. En tercer lugar, se han encontrado correlaciones significativas entre las medidas implícitas, el bienestar y los síntomas depresivos. Un mayor tiempo de reacción para asociar al Yo adjetivos que representen afectos negativos, es decir, una asociación implícita más débil entre Yo y los afectos negativos se asocia con menos síntomas depresivos y un mayor bienestar experimentado.

Por otra parte, un mayor tiempo de reacción para asociar a No Yo los afectos positivos, es decir, una asociación más débil entre No Yo y afectividad positiva, se asocia con menos síntomas depresivos y con más bienestar experimentado. Además, se ha encontrado que un mayor tiempo de reacción para asociar No Yo con afectos negativos se asocia también con menos síntomas depresivos. Finalmente, merece especial mención los resultados hallados respecto a la diferencia de las asociaciones del Yo con los afectos negativos y positivos. Dichos resultados señalaron que una mayor diferencia en la asociación del yo con los afectos negativos y positivos, es decir, una asociación más fuerte del yo con los afectos positivos y más débil con los afectos negativos, se asocia con un mayor bienestar experimentado y con menos síntomas depresivos. 
Tabla 2: Correlaciones Spearman entre las asociaciones implícitas desde el paradigma IAT y las medidas de autoinforme de depresión y felicidad subjetiva

\begin{tabular}{|c|c|c|c|c|c|c|c|c|c|c|c|c|c|c|}
\hline & 1 & 2 & 3 & 4 & 5 & 6 & 7 & 8 & 9 & 10 & 11 & 12 & 13 & 14 \\
\hline 1.Yo-Afecto Positivo & 1 & & & & & & & & & & & & & \\
\hline 2.Yo-Afecto Negativo &, $56 * * *$ & 1 & & & & & & & & & & & & \\
\hline 3.d Yo &,$- 17^{* *}$ & $64^{* * *}$ & 1 & & & & & & & & & & & \\
\hline 5. No Yo-Afecto Negativo &, $63^{* * *}$ &, $55^{* * *}$ &, $13^{*}$ &, $56 * * *$ & 1 & & & & & & & & & \\
\hline 6.d No Yo &,- 05 &, $38 * * *$ & $45^{* * *}$ &, $53^{* * *}$ &,$- 32^{* * *}$ & 1 & & & & & & & & \\
\hline 7.Síntomas depresivos &,- 11 &,$- 15^{* *}$ &,$- 12^{*}$ &,$- 18 * *$ &,$- 21^{* * *}$ & 01 & 1 & & & & & & & \\
\hline 8.Bienestar general & 07 & 03 & 03 &, 02 & 11 &,- 04 &,$- 56^{* * *}$ & 1 & & & & & & \\
\hline 10. Bienestar hedónico & 01 &,- 04 &,- 07 &,- 05 &,- 02 &,- 02 &, $14^{*}$ &, 06 &, $19 * * *$ & 1 & & & & \\
\hline 11. Bienestar social &,- 09 &,- 10 &,- 04 &,- 07 &,- 06 &,- 04 &,$- 27^{* * *}$ & $45^{* * *}$ & $37^{* * *}$ & $23^{* * *}$ & 1 & & & \\
\hline 12. Bienestar experimentado & 08 &, $16^{* *}$ &, $13^{*}$ &, $13^{*}$ &, 07 &, 06 &,$- 50 * * *$ & $46^{* * *}$ & $43^{* * *}$ &,- 10 & $25^{* \star *}$ & 1 & & \\
\hline 13. Bienestar recordado & 05 &, 04 & 02 & 02 & 08 &,- 01 &,$- 45^{* * *}$ & $83^{* * *}$ & $94^{* * *}$ & $37^{* * *}$ &, $57^{* * *}$ & $42^{* * *}$ & 1 & \\
\hline 14.Felicidad & 06 &, 06 & 04 &, 04 & 09 &,- 01 &,$- 50 * * *$ & $84^{* * *}$ & $93^{* * *}$ &, $33^{* * *}$ &, $57^{* * *}$ & $53^{* * *}$ & $99 * * *$ & 1 \\
\hline
\end{tabular}

\section{Análisis bivariantes}

Se ha encontrado que los participantes con menos niveles de depresión presentan un valor de la diferencia entre la asociación Yo-Negativo y la asociación Yo-Positivo muy superior al mostrado por los participantes que informaban de depresión severa $(M=0,63, D T=1,04$, frente a $M=0,26, D T=0,79), U=-2,47, p=0,013$. Así, los participantes sin depresión muestran una asociación mucho más fuerte del yo con los afectos positivos que con los afectos negativos. No se hallaron resultados significativos en las diferencias en las asociaciones de No Yo, ni en función del bienestar experimentado, $U=1,43, p=0,154$, ni en función de los síntomas depresivos, $U=-0,41, p=0,680$; tampoco se encontraron resultados destacados en las diferencias del Yo en función del bienestar experimentado, $U=1,57, p=0,120$. En la Figura 1 se representan gráficamente estas diferencias en las medidas implícitas en función de los niveles de síntomas depresivos y de bienestar experimentado. 


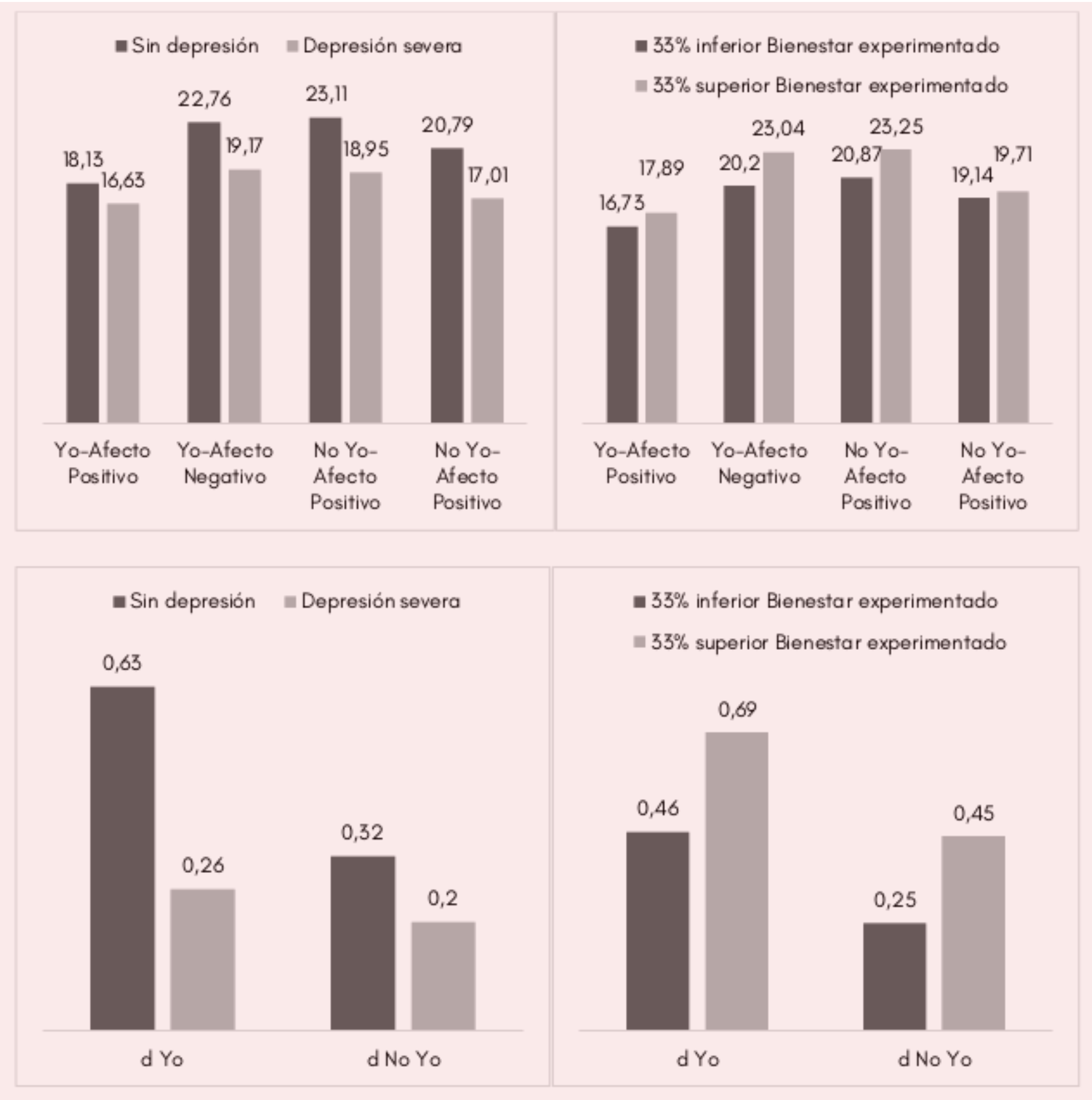

Figura 1: Tiempo de reacción en asociaciones implícitas y puntuaciones diferenciales, por niveles de síntomas depresivos (sin depresión y depresión severa) y niveles de bienestar experimentado (33\% inferior y 33\% superior).

\section{Análisis de regresión por pasos}

En estos análisis se tuvo como variables criterio el bienestar experimentado y los síntomas depresivos, y como variables predictoras las variables sociodemográficas (género, edad, estado civil, nivel de estudios, situación laboral y nivel de ingresos) y todas las medidas implícitas, tanto las asociaciones como las diferencias (ver Tabla 3). 
Tabla 3: Análisis de regresión lineal de las medidas de autoinforme de bienestar experimentado y síntomas depresivos, en base a las asociaciones implícitas y controlando las variables sociodemográficas

\begin{tabular}{|c|c|c|c|c|c|c|c|c|}
\hline \multirow{3}{*}{ Modelo } & \multicolumn{4}{|c|}{ Bienestar experimentado } & \multicolumn{4}{|c|}{ Síntomas depresivos } \\
\hline & $\Delta \mathrm{R}^{2}$ & $\mathrm{~F}$ & $t$ & $\beta$ & $\Delta R^{2}$ & $\mathrm{~F}$ & $t$ & $\beta$ \\
\hline & 05 & $2,22^{*}$ & & &, 09 & $3,51^{* * *}$ & & \\
\hline Género & & &,- 01 & 01 & & & 86 & 05 \\
\hline Edad & & & 1,29 & 08 & & & $-2,94$ &,$- 19 * *$ \\
\hline Estado civil & & &,- 55 &,- 03 & & & 30 & 02 \\
\hline Nivel académico & & & 1,60 & 10 & & & $-2,53$ &,$- 15^{*}$ \\
\hline Situación laboral & & & $-1,64$ & -10 & & & 1,40 & ,08 \\
\hline Nivel de ingreso & & & ,38 & 02 & & & 24 & ,01 \\
\hline Yo-Afecto Positivo & & &,- 57 &,- 04 & & & 09 & 01 \\
\hline Yo-Afecto Negativo & & & 3,39 & $21^{* *}$ & & &, 06 & 01 \\
\hline No Yo-Afecto Positivo & & &,- 34 &,- 04 & & & $-3,01$ &,$- 19 * *$ \\
\hline No Yo-Afecto Negativo & & & 21 & 02 & & & $-1,64$ &,- 12 \\
\hline d Yo & & &, 57 & 06 & & &,- 03 &,- 01 \\
\hline d No Yo & & &,- 38 &,- 03 & & & 1,64 &, 12 \\
\hline
\end{tabular}

Los resultados mostraron que el único predictor significativo del bienestar experimentado fue la asociación Yo - Afecto Negativo, alcanzando un porcentaje de varianza explicada de tan sólo un $5 \%$. Así, un mayor tiempo de reacción, es decir, una asociación implícita más débil a los afectos negativos, se relaciona con mayor bienestar experimentado. La prueba de DurbinWatson ofreció un valor de 1,89 por lo que los residuos no se encontraron relacionados. El gráfico de dispersión de las estimaciones tipificadas frente a los residuos tipificados no presentó ningún patrón claro, por lo que se asume igualdad de varianzas. Con respecto a los análisis de colinealidad, los datos indicaron que todos los valores de tolerancia se encontraban muy por encima de 0,10 y de FIV muy por debajo de 10, en todas las variables introducidas en el análisis de regresión.

Por su parte, los síntomas depresivos pueden ser explicados a partir de la edad, el nivel de estudios y la asociación No Yo - Afecto Positivo, explicando el $9 \%$ de la varianza. Los resultados de este análisis de regresión indicaron que una mayor edad, un mayor nivel de estudios, y un mayor tiempo de reacción en la asociación No Yo - Afecto Positivo (es decir, una asociación más débil entre No Yo y los afectos positivos), estuvo relacionado con una menor sintomatología depresiva. Igualmente, la prueba de Durbin-Watson indicó que los residuos no se encontraban relacionados ( $D W=1.90$ ), y el gráfico de dispersión no ofrecía ningún patrón, indicando homocedasticidad. Tampoco se observaron problemas de colinealidad (valores de tolerancia superiores a 0,10 y de FIV inferiores a 10 en todos los predictores). 
Con vistas a profundizar en las relaciones entre medidas implícitas y explícitas apuntadas en el análisis de regresión, se realizaron algunos análisis de varianza (ver Figura 2). En primer lugar, se crearon tres grupos en base al tiempo de reacción en la asociación entre Yo Afectos Negativos partiendo de los percentiles 33 y el 66, y se estudiaron las diferencias en bienestar experimentado entre estos grupos. La prueba Jonckheere-Terstra para muestras independientes ordenadas ha indicado diferencias significativas entre los grupos, J-T test = $2,43, p=0,015$, de manera que el grupo con un mayor tiempo de reacción en la asociación Yo-Afecto Negativo presenta una media superior en bienestar experimentado $(M=7,27, D T=$ $1,92)$ que el grupo con un menor tiempo de reacción $(M=6,64, D T=1,93)$. Así, una mayor dificultad para asociar al yo afectos negativos se asocia con un mayor bienestar experimentado. En segundo lugar, se encontraron diferencias en síntomas depresivos en función del tiempo de reacción en asociar a No Yo los afectos positivos, J-T test $=-2,97, p=$ 0,003 . El grupo con un menor tiempo de reacción presentó más síntomas depresivos $(M=$ $24,73, D T=10,59)$, en comparación con el grupo que tuvo un mayor tiempo de reacción $(M=$ $20,07, D T=6,97)$. De esta manera, una mayor facilidad para asociar a No Yo afectos positivos se asocia con más síntomas depresivos.

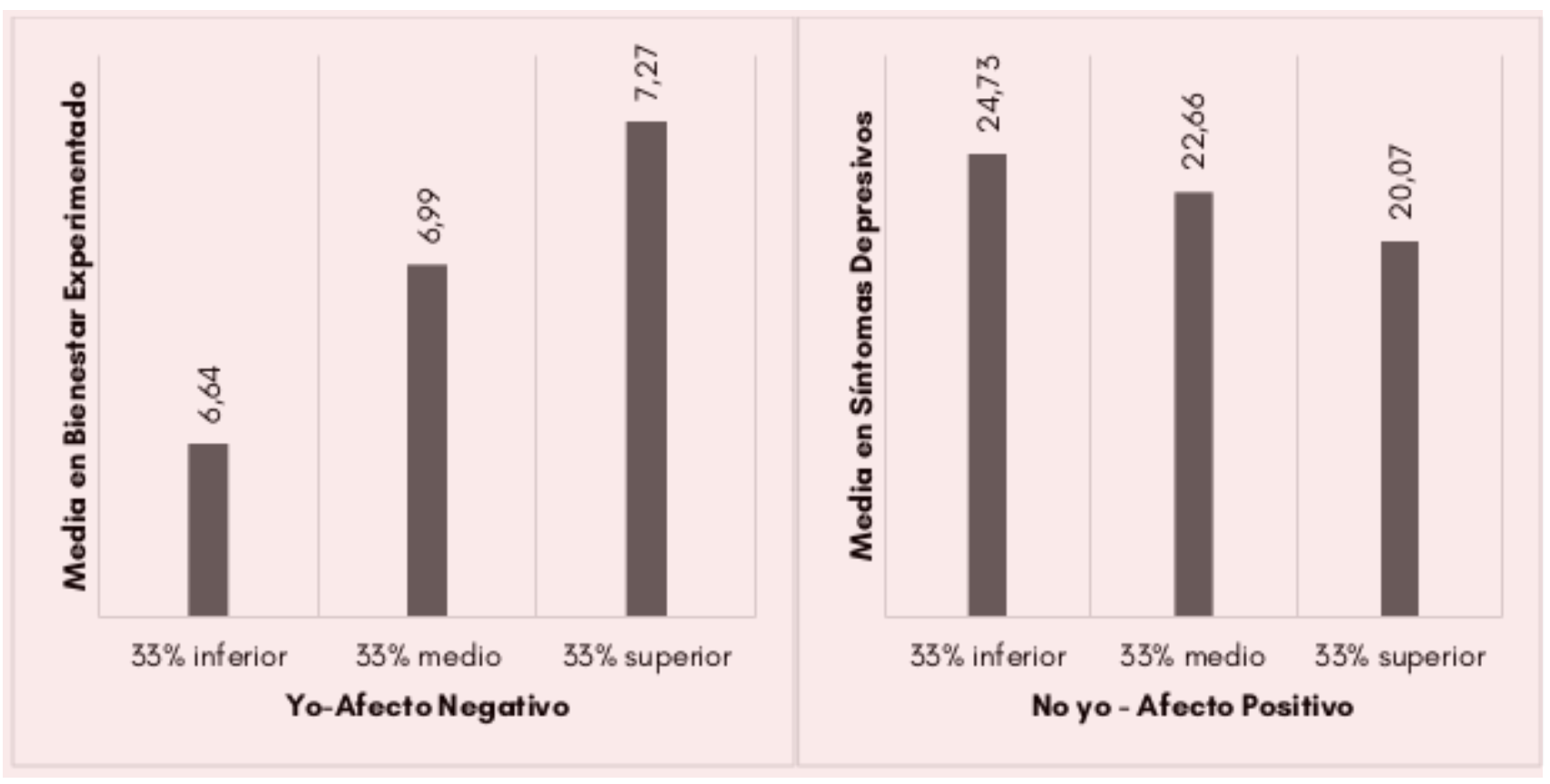

Figura 2: Puntuaciones medias en bienestar experimentado y síntomas depresivos, por niveles en las medidas implícitas en Yo - afecto negativo (33\% inferior, 33\% medio y $33 \%$ superior) y No yo - afecto positivo (33\% inferior, $33 \%$ medio y superior $33 \%$ ). 


\section{Discusión}

Tras los resultados presentados, podemos concluir que el Test de Asociación Implícita (IAT por sus siglas en inglés) que se ha presentado en esta investigación supone una vía de interés para la medición y observación del bienestar psicológico y subjetivo. En general, y acorde con estudios previos (Díaz, Horcajo y Blanco, 2009), los resultados han mostrado asociaciones pequeñas entre las medidas de IAT y ambas escalas, siendo una relación positiva con el índice de felicidad de Pemberton (PHI) y negativa con el inventario de depresión mayor (MDI). Más específicamente, un tiempo de reacción más largo en respuesta a los Afectos Negativos se asoció con menos síntomas depresivos y mayor bienestar experimentado. Por otro lado, un tiempo de reacción más largo en respuesta a los Afectos Positivos se asoció con síntomas depresivos más altos y menos bienestar experimentado. En general, una asociación implícita más fuerte con los afectos positivos que con los negativos estuvo relacionado con un mayor nivel de bienestar psicológico autoinformado. De esta forma, los resultados permiten apoyar las hipótesis de partida. Esto es, parece que el IAT diseñado y utilizado en el presente estudio logra evaluar con la fiabilidad suficiente el bienestar subjetivo de los individuos de la muestra; una muestra, por cierto, más sólida en términos de cantidad y también de heterogeneidad que la del estudio antecedente de Díaz, Horcajo y Blanco (2009). Asimismo, y corroborando también estudios previos (Díaz, Horcajo y Blanco, 2009; Walker y Schimmack (2008), se encontraron asociaciones pequeñas entre las medidas implícitas y las explícitas, tanto de felicidad como de depresión.

Por tanto, como principal contribución de la presente investigación a la observación del bienestar subjetivo o felicidad de una persona sea el diseño de un Test de Asociación Implícita (IAT) ex profeso para ello. El uso del tiempo de reacción para asociar estímulos verbales simples, de una sola palabra, parece presentar una medida del bienestar subjetivo prometedora, al evitar la mayor subjetividad y manipulatividad de una medición a través de una respuesta voluntaria y explícita con un cuestionario. Cierto es que el IAT no está exento de una manera absoluta de subjetividad, pues siempre mediará en la lectura de la palabra estímulo diferencias individuales, sociales, y también culturales (Frawley, 2015). Pero también es verdad que la incidencia de todo ello siempre será menor que en las escalas de autoinforme explícito.

Además de esto, otra cuestión que nos gustaría apuntar, y que muchos autores señalan (Bong y Lang, 2018; Kagan, 2012; White, 2017), es el hecho de que las escalas establecen explícitamente una medida de bienestar con un rango de respuestas mínimo y máximo de orden cuantitativo que no siempre es fácilmente discernible ni justificable. A lo que se agrega un problema adicional cuando se asignan etiquetas de orden cualitativo a los valores de la escala, lo cual no está exento de controversia. Así, con el IAT, las limitaciones de los rangos de respuesta se mitigan en gran medida. Ciertamente, los tiempos de reacción con un IAT también se moverán siempre entre un rango de valores, pero lo harán de manera involuntaria e implícita para el sujeto, y sin mediación o interferencia cognitiva o semántica en los valores de la propia respuesta. 
Por otro lado, medir la felicidad y el bienestar a través de IAT también presenta ventajas frente a la observación neurocientífica. Ciertamente, la neurociencia podrá permitir una observación más profunda de la actividad cerebral en múltiples procesos mentales. Podrá ser, por qué no, un camino más que interesante que recorrer en la indagación "objetiva" de la felicidad y el bienestar subjetivo y psicológico de las personas en base al comportamiento cerebral. Pero de momento sigue siendo hoy una vía cara y compleja, mientras el IAT puede representar una herramienta más económica y fácil de usar, especialmente si se quiere contar con una muestra más numerosa. Hablamos de una herramienta situada en un lugar intermedio entre las escalas y cuestionarios de autoinforme, por un lado, y por otro las mediciones que técnicas neurocientíficas, como el EEG, el eye-tracking, imágenes de resonancia magnética funcional, o la actividad electro-dérmica.

Por otro lado, sin olvidar las posibles limitaciones del tipo de muestreo y reclutamiento de la muestra, la medida IAT online permite estudios y experimentos con una muestra amplia y con un coste relativamente bajo. Así, podemos contar con una excelente muestra, en términos de alcance y heterogeneidad.

Con respecto a las limitaciones del estudio, aparte del posible inconveniente relacionado con la participación online de los sujetos, con lo que las condiciones experimentales no son igualmente controlables, conviene subrayar ciertos problemas en el uso no solo de las escalas, ya mencionados, sino también del IAT. En las respuestas explícitas obtenidas, claro está, han podido aparecer respuestas con el filtro de la deseabilidad social, el efecto techo de las escalas, la ya apuntada subjetividad en la respuesta consciente, entre otras. Pero también en la medición implícita con el IAT han podido aparecer problemas, como por ejemplo los límites fisiológicos, de percepción y psicomotores, habida cuenta de que se requería de una respuesta psicológica y también motora, con la cual el sujeto experimental no está a priori habituado. Así, podrían haber aparecido dificultades para comprender el propio procedimiento de respuesta. Además, la duración de la tarea pudo suponer cierto cansancio o aburrimiento a los participantes. Queda mucho por hacer y avanzar en relación con el uso del IAT en el estudio del ser humano, de su comportamiento y de su mente (Kurdi et al., 2019). Con todo, este trabajo pretende aportar apoyo empírico a un camino prometedor en la evaluación de la felicidad y el bienestar subjetivo y psicológico a través de procesos implícitos. Una evaluación, por tanto, menos condicionada por sesgos relacionados con la autopercepción o la manipulación consciente o inconsciente de las respuestas. Sin duda, no se trata de en ese sentido de sustituir otras vías tradicionales de evaluación psicológica, cuales son las escalas de autoinforme. Pero sí al menos para complementar estas de una manera enriquecedora con una vía de observación directa e implícita, cabría decir objetiva y más imparcial. Aun cuando estemos hablando, paradójicamente, de la observación de una experiencia tan subjetiva y relativa como es la propia felicidad de cada persona, componente crucial en esa banda interactiva acotada entre lo neurobiológico y lo sociocultural, en palabras de Muñiz (1998), que supone todo lo psicológico del ser humano. 


\section{Reconocimientos}

Este estudio fue apoyado por los fondos de investigación de la Universidad Loyola Andalucía.

\section{Conflicto de intereses}

Los autores declaran que no tienen conflicto de intereses.

\section{Cumplimiento de las normas éticas}

Aprobación ética: Todos los procedimientos realizados en estudios con participantes humanos se ajustaron a las normas éticas del comité de investigación institucional y / o nacional y a la declaración de Helsinki de 1964 y sus modificaciones posteriores o normas éticas comparables. Se obtuvo el consentimiento informado de todos los participantes individuales incluidos en el estudio.

\section{Referencias}

Alexander, R., Aragon, O. R., Bookwala, J., Cherbuin, N., Gatt, J. M., Kahrilas, I. J., ... Styliadis, C. (2021). The neuroscience of positive emotions and affect: Implications for cultivating happiness and wellbeing. Neuroscience and Biobehavioral Reviews, 121, 220-249. https://doi.org/10.1016/j.neubiorev.2020.12.002

Bech, P., Rasmussen, N.-A., Olsen, L. R., Noerholm, V., y Abildgaard, W. (2001). The sensitivity and specificity of the Major Depression Inventory, using the Present State Examination as the index of diagnostic validity. Journal of Affective Disorders, 66(2), 159-164. https://doi.org/10.1016/S0165-0327(00)00309-8

Bond, T. N., y Lang, K. (2018). The Sad Truth About Happiness Scales. Journal of Political Economy. https://doi.org/10.1086/701679

Briñol, P., Horcajo, J., Becerra, A., Falces, C., y Sierra, B. (2002). Cambio de actitudes implícitas. Psicothema, 14(4), 771-775. https://tinyurl.com/ryhtyy87

Burgess, L., Nugent, S., Hunt, G., y Eslami, A. (2019). Pilot study: youth suicide assessment by lmplicit Association Testing (IAT) in emergency psychiatry. Journal of the American Academy of Child and Adolescent Psychiatry, 58(10, S), S213-S214. https://doi.org/10.1016/j.jaac .2019.08.221

Byrne, B. M. (2013). Structural equation modeling with AMOS: Basic concepts, applications, and programming. Routledge

Caputo, A. (2017). Social desirability bias in self-reported well-being measures: Evidence from an online survey. Universitas Psychologica, 16(2), 1-13. https://doi.org/10.11144/Javeriana. upsy16-2.sdsw

Cárdenas, M., y Barrientos, J. (2008). Actitudes explícitas e implícitas hacia los hombres homosexuales en una muestra de estudiantes universitarios en Chile. Psykhe (Santiago), 17(2), 17-25. https://doi.org/10.4067/S0718-22282008000200002 
Cárdenas, M., González, C., Calderón, C., y Lay, S. L. (2009). Medidas explícitas e implícitas de las actitudes hacia las mujeres. Revista Interamericana de Psicología/Interamerican Journal of Psychology, 43(3), 541-546. https://tinyurl.com/4xmax6wt

Corral-Frías, V., Lucas, M., Corral-Frias, N., Corral-Verdugo, V., y Tapia, C. (2019). Assessment of Affinity towards Diversity Using the Implicit Association Test and Self-Reports. Sustainability, 11, 5825. https://doi.org/10.3390/sul1205825

Costantini, G., Perugini, M., Dentale, F., Barbaranelli, C., Alessandri, G., Vecchione, M., y Caprara, G. V. (2019). Assessing Positive Orientation with the Implicit Association Test. European Journal of Psychological Assessment, 35, 109-116. https://doi.org/10.1027/1015$5759 / a 000362$

Da Victoria, M. S., Nascimento, A. L., y Fontenelle, L. (2011). Selection of visual stimuli for the Implicit Association Test for the Obsessive-Compulsive Disorder (IAT-OCD). Revista de Psiquiatria Clinica, 38(3), 102-105. https://doi.org/10.1590/S0101-60832011000300004

De las Cuevas y Catresana, C., y González de Rivera y Revuelta, J. (1992). Autoinformes y respuestas sesgadas. Anales de Psiquiatría, 8(9), 362 -366. https://tinyurl.com/5afjkn7w

Denegri, C. M., García, J. C., y González, R. N. (2015). Definición de bienestar subjetivo en adultos jóvenes profesionales chilenos. Un estudio con redes semánticas naturales. CES Psicología, 8(1), 77-97. https://doi.org/10.21615/3216

Deng, X., Ding, X., Cheng, C. y Chou, H. M. (2016). Feeling Happy and Sad at the Same Time? Subcultural Differences in Experiencing Mixed Emotions between Han Chinese and Mongolian Chinese. Frontiers in Psychology, 7. https://doi.org/10.3389/fpsyg.2016.01692

Díaz, D., Horcajo, J., y Blanco, A. (2009). Development of an Implicit Overall Well-Being Measure Using the Implicit Association Test. The Spanish Journal of Psychology, 12(2), 604-617. https://doi.org/10.1017/S1138741600001979

Diener, E. (1994). Assessing subjective well-being: Progress and opportunities. Social Indicators Research, 31, 103-157. https://doi.org/10.1007/BF01207052

Diener, E., Wirtz, D., Tov, W., Kim-Prieto, C., Choi, D., Oishi, S., y Biswas-Diener, R. (2009). New measures of well-being: Flourishing and positive and negative feelings. En E. Diener (Ed.), Assessing Well-Being (pp. 247-266), Springer. https://doi.org/10.1007/978-90-481-23544_12

Diener, E., Horwitz, J., y Emmons, R. A. (1985). Happiness of the very wealthy. Social Indicators Research, 16, 263-274 https://doi.org/10.1007/BF00415126

Diener, E., Sandvik, E., Pavot, W., y Gallagher, D. (1991). Response artifacts in the measurement of subjective well-being. Social Indicators Research, 24(1), 35-56. https://doi.org/ 10.1007/BF00292649 
Flores-Kanter, P.E., Muñoz-Navarro, R. y Medrano, L. A. (2018) Concepciones de la Felicidad y su relación con el Bienestar Subjetivo: un estudio mediante Redes Semánticas Naturales. Liberabit, 24(1), 115-130 https://doi.org/10.24265/liberabit.2018.v24n1.08

Fordyce, M. W. (1988). A Review of research on the happiness measures: A sixty second index of happiness and mental health. Social Indicators Research, 20, 355-381. https://doi.org/10.1007/BF00302333

Frawley, A. (2015) Happiness Research: A Review of Critiques. Sociology Compass, 9, 62- 77, https://doi.org/10.1111/soc4.12236

Garín, M., Huenchuleo, J., Leal, N., Muñoz, A. M., y Rehbein, L. (2013). Actitudes implícitas hacia la violencia, conducta antisocial y consumo televisivo en estudiantes universitarios. Revista de Psicología, 22(2), 100-110. https://doi.org/10.5354/0719-0581.2013.30857

Gómez-Benito, J., Hidalgo, M. D., y Guilera, G. (2010). El sesgo de los instrumentos de medición. Test justos. Papeles del Psicólogo, 3(1), 75-84. https://tinyurl.com/yjteftwy

Greenwald, A. G., y Farnham, S. D. (2000). Using the implicit association test to measure selfesteem and self-concept. Journal of Personality and Social Psychology, 79(6), 1022-1038. https://doi.org/10.1037/0022-3514.79.6.1022

Greenwald, A. G., Nosek, B. A., y Banaji, M. R. (2003). Understanding and using the implicit association test: I. An improved scoring algorithm. Journal of Personality and Social Psychology, 85(2), 197-216. https://doi.org/10.1037/0022-3514.85.2.197

Greenwald, A. G., Poehlman, T. A., Uhlmann, E. L., y Banaji, M. R. (2009). Understanding and using the Implicit Association Test: III. Meta-analysis of predictive validity. Journal of personality and social psychology, 97(1), 17-41. https://doi.org/10.1037/a0015575

Hervás, G., y Vázquez, C. (2013). Construction and validation of a measure of integrative wellbeing in seven languages: The Pemberton Happiness Index. Health and quality of life outcomes, 17(1), 66-79, https://doi.org/10.1186/1477-7525-11-66

Hills, P., y Argyle, M. (2002). The Oxford Happiness Questionnaire: A compact scale for the measurement of psychological well-being. Personality and Individual Differences, 33(7), 1071-1082. https://doi.org/10.1016/S0191-8869(01)00213-6

Hofmann, W., Gawronski, B., Gschwendner, T., Le, H., y Schmitt, M. (2005). A meta-analysis on the correlation between the Implicit Association Test and explicit self-report measures. Personality and Social Psychology Bulletin, 37(10), 1369-1385. https://doi.org/10.1177/ 0146167205275613 
Horcajo, J., Rubio, V. J., Aguado, D., Hernández, J. M. y Márquez, M. O. (2014). Using the Implicit Association Test to Assess Risk Propensity Self-concept: Analysis of its Predictive Validity on a Risk-taking Behaviour in a Natural Setting, European Journal of Personality, 28, 459-471. https://doi.org/10.1002/per.1925

Hu, L. T., y Bentler, P. M. (1999). Cutoff criteria for fit indexes in covariance structure analysis: Conventional criteria versus new alternatives. Structural equation modeling: a multidisciplinary journal, 6(1), 1-55. https://doi.org/10.1080/10705519909540118

Irving, L. H., y Smith, C. T. (2020). Measure what you are trying to predict: Applying the correspondence principle to the Implicit Association Test. Journal of Experimental Social Psychology, 86, 103898. https://doi.org/10.1016/j.jesp.2019.103898

Jost, J. T. (2019). The IAT Is Dead, Long Live the IAT: Context-Sensitive Measures of Implicit Attitudes Are Indispensable to Social and Political Psychology. Current Directions in Psychological Science, 28(1), 10-19. https://doi.org/10.1177/0963721418797309

Kagan, J. (2012). Psychology's Ghosts: The Crisis in the Profession and the Way Back. Yale University Press.

Kahneman, D., Krueger, A. B., Schkade, D. A., Schwarz, N., y Stone, A. A. (2004). A Survey Method for Characterizing Daily Life Experience: The Day Reconstruction Method. Science, 306(5702), 1776-1780. https://doi.org/10.1126/science.1103572

Kurdi, B., Seitchik, A. E., Axt, J. R., Carroll, T. J., Karapetyan, A., Kaushik, N., Tomezsko, D., Greenwald, A. G., y Banaji, M. R. (2019). Relationship between the Implicit Association Test and intergroup behavior: A meta-analysis. American Psychologist, 74(5), 569-586. https://doi.org/10.1037/amp0000364

Lyubomirsky, S., y Lepper, H. (1999). A Measure of Subjective Happiness: Preliminary Reliability and Construct Validation. Social Indicators Research, 46, 137-155. https://doi.org/ 10.1023/A:1006824100041

Muñiz, J. (1998). La medición de lo psicológico. Psicothema, 10 (1), 1-21. https://tinyurl.com/ kykvx9m

Muñiz-Velázquez, J. A., Gomez-Baya, D. y Lopez-Casquete, M. (2017). Implicit and explicit assessment of materialism: Associations with happiness and depression. Personality and Individual Differences, 116, 123-132. https://doi.org/10.1016/j.paid.2017.04.033

Nebreda, C., y Aliaga, L. (2003). Major Depression Inventory-MDI. Hillerød: Psychiatric Research Unit. Mental Health Centre North Zealand. 
Nosek, B. A., Greenwald, A. G., Banaji, M. R. (2005). Understanding and using the Implicit Association Test: II. Method variables and construct validity. Personality and Social Psychology Bulletin, 31(2), 166-180. https://doi.org/10.1177/0146167204271418

Olivos, P., y Aragonés, J. I. (2013). Test de asociaciones implícitas con la naturaleza: aplicaci ón en España del "IAT-Nature". Revista de Psicología Social, 28(2), 237-245. https://doi.org/10.1174/021347413806196672

Olsen, L. R., Jensen, D. V., Noerholm, V., Martiny, K., y Bech, P. (2003). The internal and external validity of the Major Depression Inventory inmeasuring severity of depressive states. Psychological Medicine, 33(2), 351-356. https://doi.org/10.1017/S0033291702006724

Park, J., Uchida, Y. y Kitayama, S. (2016). Cultural variation in implicit independence: An extension of Kitayama et al. International Journal of Psychology, 51, 269-278. https://doi.org/ 10.1002/ijop.1215

Peterson, C. (2005). Authentic Happiness Inventory. https://tinyurl.com/uabuv25m

Rath, D., Hallensleben, N., Glaesmer, H., Spangenberg, L., Strauss, M., Kersting, A., ... Forkmann, T. (2018). Implicit Associations with Death: First Validation of the German Version of the Suicide Implicit Association Test (Suicide IAT). Psychotherapie Psychosomatik Medizinische Psychologie, 68(3-4), 109-117. https://doi.org/10.1055/s-0043-105070

Riediger, M., Wrzus, C., y Wagner, G. G. (2014). Happiness is pleasant, or is it? Implicit representations of affect valence are associated with contrahedonic motivation and mixed affect in daily life. Emotion, 14, 950-961. https://doi.org/10.1037/a0037711

Rohner, J., y Ewers, T. (2016). Trying to separate the wheat from the chaff: Construct-and faking-related variance on the Implicit Association Test (IAT). Behavior Research Methods, 48(1), 243-258. https://doi.org/10.3758/s13428-015-0568-1

Sánchez, A. A. (2012). La utilización del Test de Asociación Implícita en los procesos electorales. Revista Justicia Electoral, 1(10), 267-292. https://tinyurl.com/usdyyu5j

Sanchez, A., Vazquez, C., Gomez, D., y Joormann, J. (2014). Gaze-fixation to happy faces predicts mood repair after a negative mood induction. Emotion, 14(1), 85-94. https://doi.org/10.1037/a0034500

Salgado-Montejo, A., Salgado, C. J., Alvarado, J., y Spence, C. (2017). Simple lines and shapes are associated with, and communicate, distinct emotions. Cognition and Emotion, 31(3), https://doi.org/10.1080/02699931.2015.1133401

Sriram, N., y Greenwald, A. G. (2009). The brief implicit association test. Experimental psychology, 56(4), 283-294. https://doi.org/10.1027/1618-3169.56.4.283 
Tennant, R., Hiller, L., Fishwick, R., Platt, S., Joseph, S., Weich, S., ... ,Stewart-Brown, S. (2007). The Warwick-Edinburgh Mental Well-being Scale (WEMWBS): development and UK validation. Health and Quality of Life Outcomes, 5(1), 63. https://doi.org/10.1186/14777525-5-63

Van de Mortel, T. F. (2008). Faking it: Social desirability response bias in self-report research. Australian Journal of Advanced Nursing, 25(4), 40-48.

Walker, S. S., y Schimmack, U. (2008). Validity of a happiness Implicit Association Test as a measure of subjective well-being. Journal of Research in Personality, 42(2), 490-497. https://doi.org/10.1016/j.jrp.2007.07.005

Warriner, A. B., Shore, D. I., Schmidt, L. A., Imbault, C. L., y Kuperman, V. (2017). Sliding into happiness: A new tool for measuring affective responses to words. Canadian Journal of Experimental Psychology/Revue canadienne de psychologie expérimentale, 71(1), 71-88. https://doi.org/10.1037/cep0000112

Watson, D., Clark, L. A., y Tellegen, A. (1988). Development and validation of brief measures of positive and negative affect: The PANAS scales. Journal of Psychology, 54(6), 1063-1070. https://doi.org/10.1037/0022-3514.54.6.1063

White, M. D. (2017). Judging the Efficacy and Ethics of Positive Psychology for Government Policymaking. En T. Lomas, N. Brown, y F. J. Eiroá-Orosa (Eds.), The Routledge International Handbook of Critical Positive Psychology (pp. 532-545). Routledge. https://doi.org/ 10.4324/9781315659794.ch35

Young, K., Kashdan, T., y Macatee, R. (2014). Strength balance and implicit strength measurement: New considerations for research on strengths of character. The Journal of Positive Psychology, 10, 17-24. https://doi.org/10.1080/17439760.2014.920406

Zevnik, L. (2014). The Discussion about the Universality of Happiness and the Promise of Neuroscience. Cultura-International Journal of Philosophy of Culture and Axiology, 11(1), 41-62. https://doi.org/10.5840/cultura20141113

\section{Para citar en APA}

Gómez-Baya, D. y Muñiz-Velázquez, J. A. (2021). La medición de la felicidad a través del Test de Asociación Implícita y su relación con la depresión y el bienestar autoinformados. Terapia Psicologíca (En línea), 39(1), 39-61. https://doi.org/10.4067/S0718-48082021000100039 